\title{
ICTIOPLANCTON EN LA CIÉNAGA DE AYAPEL, RÍO SAN JORGE (COLOMBIA): CAMBIOS ESPACIO-TEMPORALES*
}

\author{
Luz Eneida Ochoa-Orrego ${ }^{1}$, Luz Fernanda Jiménez ${ }^{2}$, Jaime Palacio ${ }^{3}$
}

\section{Resumen}

Muestreos de ictioplancton fueron realizados en ocho sitios en la Ciénaga de Ayapel a lo largo del ciclo hidrológico del río San Jorge. Se utilizó una red cónica con diámetro de abertura de $35 \mathrm{~cm}$ con flujómetro instalado en la boca de la red, para la obtención del volumen de agua filtrada. Las larvas fueron agrupadas según su orden taxonómico y fase de desarrollo. Se capturaron en total 1321 individuos, agrupados en 6 especies y 27 morfotipos. Las densidades oscilaron entre 0,29 y 576 individuos $/ \mathrm{m}^{-3}$. Los grupos de siluriformes y characiformes fueron los que presentaron las mayores densidades, las cuales estuvieron asociadas con factores físicos como las lluvias, el nivel del agua de la ciénaga y el pulso de inundación del río San Jorge. Este último factor es responsable de la inundación de las áreas laterales generando un aumento de nutrientes y microhábitats, que ofrecen alimento y refugio para el desarrollo de los individuos en sus periodos iniciales de desarrollo ontogénico.

Palabras clave: ictioplancton, lago de inundación, río tropical, río San Jorge.

\section{ICTHYOPLANKTON IN THE AYAPEL FLOODPLAIN LAKE, SAN JORGE RIVER (COLOMBIA): SPATIAL AND TIME CHANGES}

\begin{abstract}
Ichthyoplankton surveys were conducted in eight sampling sites in Ayapel Floodplain Lake during a one hydrological periods. We used a conical net with a diameter of $35 \mathrm{~cm}$ opening with a flowmeter installed at the mouth of the net to obtain the volume of filtered water. The larvae were grouped according to their taxonomic order and development phase. We captured 1321 individuals grouped into 6 species and 27 morphotypes. The densities were between 0.29 and 576 individuals $/ \mathrm{m}^{-3}$. Sampling sites near to the San Jorge River influence showed the highest densities. Silurifoms and Characiforms were the most important fish orders in the samples and their densities were associated with the physical factors such as rains, water level, the conductivity in the lake and the floods of the San Jorge River. The last factor was responsible for the flooding of lateral areas, which leads to increase nutrients and microhabitats that provide food and shelter for the development of individuals in the early periods of its ontogenic development.
\end{abstract}

Key words: ichthyoplankton, floodplain lake, tropical river, San Jorge River.

\footnotetext{
${ }^{*}$ FR: 10-II-2014, . FA: .22-XI-2014

${ }^{1}$ Instituto de Biología, Universidad de Antioquia. Medellín, Colombia. E-mail: luzeocho@gmail.com

${ }^{2}$ Instituto de Biología, Universidad de Antioquia. Medellín, Colombia. E-mail: luz.jimenez@udea.edu.co

3 Departamento de Ingeniería Sanitaria, Grupo de Investigación y Modelación Ambiental (GAIA), sede de Investigaciones Universitaria SIU. Medellín, Colombia. E-mail: japalaci@udea.edu.co 


\section{INTRODUCCIÓN}

Los estudios sobre distribución y abundancia de ictioplancton son de extrema importancia para la determinación de periodos y lugares de desove, tornándose fundamentales tanto para la taxonomía como para la ecología de las especies, ya que la información sobre una comunidad íctica, no puede ser considerada adecuada sin un buen conocimiento de la ecología de los periodos iniciales del ciclo de vida. En las últimas décadas se ha observado un interés creciente en estos estudios, principalmente en la ocurrencia e identificación de áreas de reproducción y crecimiento, que constituyen un dato valioso para el aprovechamiento de los recursos hídricos, ayudando tanto a la ictiología como a la biología pesquera (BIALETZKI et al., 1999; ARAUJO-LIMA et al., 2001; BIALETZKI et al., 2002; JIMÉNEZ-SEGURA, 2007).

De estudios enfocados en la dinámica del ictioplancton en ríos en América Latina, pueden citarse los trabajos de ARAUJO-LIMA \& OLIVEIRA (1998) para el Amazonas, en el embalse de Itaipú en la cuenca alta del río Paraná (NAKATANI et al., 1993), en la cuenca baja del río Paraná (OLDANI., 1990; OLDANI et al., 1992), en el delta del río Paraná (FUENTES \& ESPINACH ROS, 1998; BIALETZKI et al., 1999; GONZÁLEZ et al., 2010) y en zonas de inundación de este río principalmente en lagos marginales con diferentes grados de conectividad (BAUMGARTNER et al., 2004, 2008; SALETE et al., 2009), en el río Ivinhema en Brasil (REYNALTE-TATAJE et al., 2011) y en el río Magdalena (JIMÉNEZ-SEGURA, 2007; PAREJA-CARMONA et al., 2014).

En ríos tropicales con planicie de inundación, la reproducción de los peces que migran durante el estiaje ocurre en la época de crecientes (WELCOMME, 1979; ARAUJO-LIMA, 1994). Los eventos de desove durante las crecientes, reducen la depredación intra e ínter-específica (LUCAS \& BARAS, 2001) y aseguran el transporte de la prole hasta las áreas de crianza en los planos de inundación donde encuentran refugio y alimento (LOWE-McCONNELL, 1987). El ingreso de ictioplancton a los lagos del plano lateral de estos sistemas esta determinado por la influencia del río principal y la temporalidad de sus crecientes. De acuerdo con WELCOMME (1985) a medida que el caudal de un río y por tanto el nivel del agua se incrementa, el canal reduce su capacidad de tránsito, el agua se desborda sobre su plano lateral, permitiendo la conexión entre estos dos ambientes (río y ciénaga lago). Se ha observado que la distribución de las larvas de peces y otros grupos de organismos que hacen parte del zooplancton está dada por la respuesta diferencial de los organismos a procesos hidrodinámicos y biológicos, que presentan variación a escala espacial y temporal (MACKAS et al., 1985). Las larvas de peces muestran patrones de distribución relacionados con la variabilidad hidrográfica (LEGENDRE \& DEMERS, 1984). En este sentido, factores como los cambios en el nivel del agua y parámetros como la conductividad son considerados claves en los patrones de distribución horizontal.

Colombia actualmente presenta cerca de 20’252.500 de hectáreas de humedales (17,48\% de su territorio continental) representados por el 52\% en ciénagas, $27 \%$ en lagunas, $8 \%$ en esteros, $6 \%$ en páramos, $4 \%$ son marinos, $2 \%$ son embalses y el $1 \%$ lagos, los cuales proveen múltiples bienes y servicios para el desarrollo de las actividades económicas de las comunidades locales, así como para la conservación de la biota que albergan (MINISTERIO DEL MEDIO AMBIENTE \& IiAvH, 1999; NARANJO Y PONCE, 1999). Los humedales, ubicados en las zonas laterales de los ríos, son considerados áreas altamente productivas puesto que los aportes que hacen los ríos durante los periodos de crecientes, se incorporan rápidamente en 
biomasa (WINEMILLER, 1998), dinamizan el flujo de carbono dentro de la cuenca (KONDOLF et al., 2006) y son vitales para la productividad pesquera de la cuenca del río Magdalena.

El presente trabajo tiene como objeto conocer la distribución espacial y temporal del ictioplancton dentro de un sistema cenagoso colombiano y brindar información sobre la abundancia de taxones, así como de la interacción de factores abióticos en la distribución del ictioplancton en la planicie inundable.

\section{METODOLOGÍA}

Las muestras de ictioplancton fueron tomadas durante un periodo hidrológico completo, en ocho estaciones de muestreo (Tabla 1) ubicadas en la Ciénaga de Ayapel (Ayapel, Córdoba), las cuales comprenden cuatro (4) pequeñas ciénagas y tres (3) caños de conexión con el río San Jorge los cuales fueron muestreados por ser sitios de conexión con el canal principal del río.

Para el muestreo se empleó una red cónica con diámetro de abertura de $35 \mathrm{~cm}$, con un flujómetro General Oceanics instalado en la boca de la red. El material fue fijado en formol carbonatado al $2 \%$ y llevado al laboratorio para su procesamiento.

De cada muestra fue separado el ictioplancton (huevos, embriones y larvas) del material orgánico e inorgánico acompañante, utilizando un estereoscopio Olympus CH30. Las larvas fueron agrupadas según su taxa y fase de desarrollo. Las fases de desarrollo utilizadas siguieron la propuesta de KENDALL et al. (1984), modificada por NAKATANI et al. (2001). La identificación de especies y morfotipos se realizó siguiendo la clave preliminar para larvas del río Magdalena (JIMÉNEZ-SEGURA, 2007).

La densidad de individuos fue calculada para $1 \mathrm{~m}^{3}$ de acuerdo con la siguiente expresión: $\mathrm{Y}=(\mathrm{X} / \mathrm{V})$, donde $\mathrm{Y}=$ densidad de huevos y larvas $/ \mathrm{m}^{3}, \mathrm{X}=$ número de huevos y larvas capturados y $\mathrm{V}=$ volumen de agua filtrada $\left(\mathrm{m}^{3}\right)$. El volumen de agua filtrada por la red fue calculado de acuerdo al factor de calibración del flujómetro ajustado por el diámetro de la boca de la red. Datos de conductividad y nivel del agua fueron registrados en cada una de las estaciones de muestreo.

Tabla 1. Ubicación geográfica de las estaciones de muestreo.

\begin{tabular}{lccc}
\hline \multirow{2}{*}{ Estación de muestreo } & \multirow{2}{*}{ Sigla } & \multicolumn{2}{c}{ Coordenadas } \\
\cline { 3 - 4 } & & N & W \\
\hline Ciénaga Río & CNGR & $08^{\circ} 26^{\prime} 05,7^{\prime \prime}$ & $75^{\circ} 03^{\prime} 40^{\prime \prime}$ \\
Ciénaga Transición & CNGT & $08^{\circ} 20^{\prime} 02,4^{\prime \prime}$ & $75^{\circ} 05^{\prime} 25,2^{\prime \prime}$ \\
Ciénaga Paticos & CNGP & $08^{\circ} 19^{\prime} 39^{\prime \prime}$ & $75^{\circ} 08^{\prime} 31,9^{\prime \prime}$ \\
Ciénaga Mercado & CNGM & $08^{\circ} 18^{\prime} 30,5^{\prime \prime}$ & $75^{\circ} 08^{\prime} 27,9^{\prime \prime}$ \\
Caño Barro & CB & $08^{\circ} 17^{\prime} 5,6^{\prime \prime}$ & $75^{\circ} 03^{\prime} 46,3^{\prime \prime}$ \\
Caño Grande & CG & $08^{\circ} 27^{\prime} 28,6^{\prime \prime}$ & $75^{\circ} 02^{\prime} 54,0^{\prime \prime}$ \\
Cańo Muńoz & CM & $08^{\circ} 20^{\prime} 25,1^{\prime \prime}$ & $74^{\circ} 56^{\prime} 31,4^{\prime \prime}$ \\
Quebrada Quebradona & QQ & $08^{\circ} 17^{\prime} 19^{\prime \prime}$ & $75^{\circ} 09^{\prime} 25^{\prime \prime}$ \\
\hline
\end{tabular}




\section{Área de estudio}

El estudio se realizó en la Ciénaga de Ayapel (Figura 1) ubicada en el departamento de Córdoba, en una zona de bosque húmedo tropical, con temperaturas mínimas de $26^{\circ} \mathrm{C}$ y máximas de $28,7^{\circ} \mathrm{C}$ (IGAC, 1986). El régimen de precipitaciones en la región de Ayapel presenta en general una época de lluvias de abril a noviembre y una seca de diciembre a marzo. El promedio anual de lluvias fluctúa entre 2000 y $2500 \mathrm{~m}$. Este sistema está asociado a la llanura aluvial del río San Jorge (IGAC, 1986), y es quizá una de las ciénagas más grandes de la región de La Mojana (RÍOS, 2006).

Se caracteriza por ser un humedal raso de espejo de agua permanente, con una profundidad máxima cercana a los $6 \mathrm{~m}$. Tiene una extensión aproximada de $45 \mathrm{~km}^{2} \mathrm{y}$ posee un sistema hidrológico propio (independiente) que recoge las precipitaciones de las quebradas de los costados sur y oriental. El sistema entrega sus excedentes al río San Jorge por los caños Grande y Victoria, y mantiene la regulación de los niveles altos del mismo río a través de estos (RESTREPO, 2005).

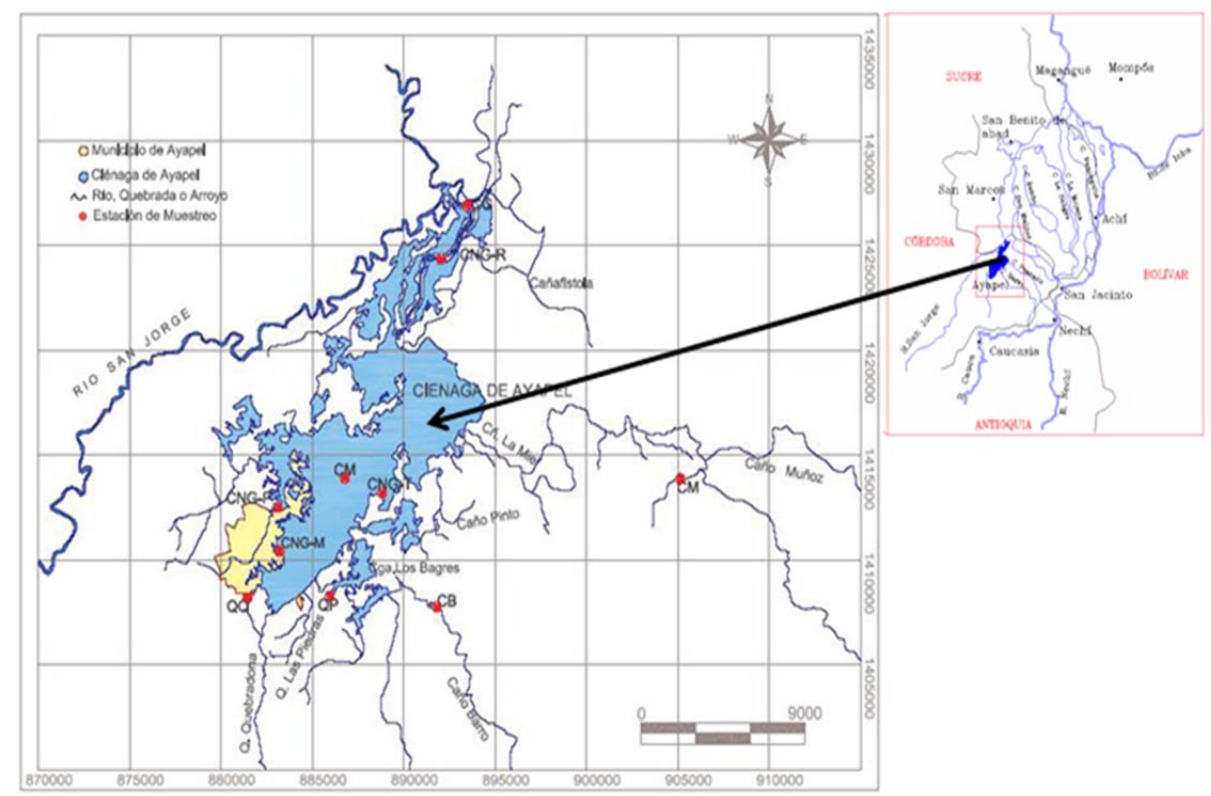

Figura 1. Ubicación de la Ciénaga de Ayapel en el departamento de Córdoba (Colombia) y las estaciones de muestreo.

\section{RESULTADOS}

Se colectaron en total 1318 individuos, agrupados en 7 especies y 26 morfotipos (Tabla 2). La especie más abundante correspondió a Astyanax sp. en fase larvalvitelino y preflexión, seguido de Pimelodus blochii en fase de flexión, "characiforme 7" y "characiforme 4" en fase larval-viltelino. 
Tabla 2. Especies y morfotipos identificados por fase de desarrollo larval. (n) número de individuos, (FO) frecuencia de ocurrencia, y densidad en individuos por $\mathrm{m}^{3}$.

\begin{tabular}{|c|c|c|c|c|c|}
\hline Orden & Especie/Morfotipo & Fase & $\mathbf{n}$ & FO & Densidad ind $/ \mathrm{m}^{3}$ \\
\hline \multirow{8}{*}{ Siluriformes } & Sorubim cuspicaudus & Prefexión & 5 & 0,004 & 0,580 \\
\hline & Pimelodus blochii & $\begin{array}{l}\text { Preflexión } \\
\text { Flexión }\end{array}$ & $\begin{array}{c}22 \\
257\end{array}$ & $\begin{array}{l}0,017 \\
0,195\end{array}$ & 32,290 \\
\hline & Pseudoplatystoma & Preflexión & 8 & 0,006 & \multirow{2}{*}{2,570} \\
\hline & magdaleniatum & Flexión & 19 & 0,014 & \\
\hline & Siluriforme 10 & Preflexión & 15 & 0,011 & 2,090 \\
\hline & \multirow{2}{*}{ Siluriforme 13} & Flexión & 3 & 0,002 & \multirow{2}{*}{0,040} \\
\hline & & Posflexión & 1 & 0,001 & \\
\hline & Siluriforme 14 & Preflexión & 1 & 0,001 & 0,040 \\
\hline \multirow{20}{*}{ Characiformes } & Prochilodus magdalenae & Larval-vitelino & 7 & 0,005 & \multirow{2}{*}{2,290} \\
\hline & & Preflexión & 17 & 0,013 & \\
\hline & Leporinus muyscorum & Preflexión & 2 & 0,002 & 0,180 \\
\hline & \multirow{2}{*}{ Astyanax sp. } & Larval-vitelino & 312 & 0,237 & \multirow{2}{*}{57,040} \\
\hline & & Preflexión & 158 & 0,120 & \\
\hline & Characiforme 8 & Preflexion & 2 & 0,002 & 0,110 \\
\hline & Characiforme 10 & Preflexión & 3 & 0,002 & 0,080 \\
\hline & Characiforme 4 & Larval-vitelino & 129 & 0,098 & 20,940 \\
\hline & Characiforme 7 & $\begin{array}{l}\text { Preflexión } \\
\text { Larval-vitelino }\end{array}$ & $\begin{array}{c}61 \\
198\end{array}$ & $\begin{array}{l}0,046 \\
0,150\end{array}$ & 22,960 \\
\hline & Characiforme 11 & Larval-vitelino & 5 & 0,004 & 0,230 \\
\hline & \multirow[t]{2}{*}{ Characiforme 12} & Preflexión & 5 & 0,004 & \multirow{2}{*}{0,110} \\
\hline & & Posflexión & 2 & 0,002 & \\
\hline & Characiforme 16 & $\begin{array}{l}\text { Larval-ví́inno } \\
\text { Preflexión }\end{array}$ & 14 & $\begin{array}{l}0,005 \\
0,011\end{array}$ & $\begin{array}{l}0,810 \\
0,870\end{array}$ \\
\hline & Characiforme 17 & Flexión & 4 & 0,003 & 0,410 \\
\hline & Characiforme 21 & Preflexión & 4 & 0,003 & 0,470 \\
\hline & Characiforme 22 & Preflexión & 3 & 0,002 & 0,160 \\
\hline & Characiforme 23 & Preflexión & 1 & 0,001 & 0,120 \\
\hline & Characiforme 28 & preflexión & 1 & 0,001 & 0,120 \\
\hline & Characiforme 42 & Preflexión & 1 & 0,001 & 0,050 \\
\hline & Characiforme 44 & Preflexión & 1 & 0,001 & 0,020 \\
\hline Perciformes & $\begin{array}{l}\text { Plagioscion } \\
\text { surinamensis }\end{array}$ & Preflexión & 22 & 0,017 & 0,400 \\
\hline \multirow{8}{*}{$\begin{array}{l}\text { No } \\
\text { determinados }\end{array}$} & LV 4 & Larval-vitelino & 3 & 0,002 & 0,350 \\
\hline & LV 7 & Larval-vitelino & 1 & 0,001 & 0,040 \\
\hline & LV 8 & Larval-vitelino & 4 & 0,003 & 0,160 \\
\hline & LV 9 & Larval-vitelino & 1 & 0,001 & 0,120 \\
\hline & LV 10 & Larval-vitelino & 8 & 0,006 & 1,230 \\
\hline & LV 11 & Larval-vitelino & 2 & 0,002 & 0,230 \\
\hline & LV 14 & Larval-vitelino & 1 & 0,001 & 0,120 \\
\hline & Huevos & Clivaje & 8 & 0,006 & 0,340 \\
\hline
\end{tabular}

Los characiformes fueron los más abundantes tanto en individuos como en especies y morfotipos (Figura 2). 


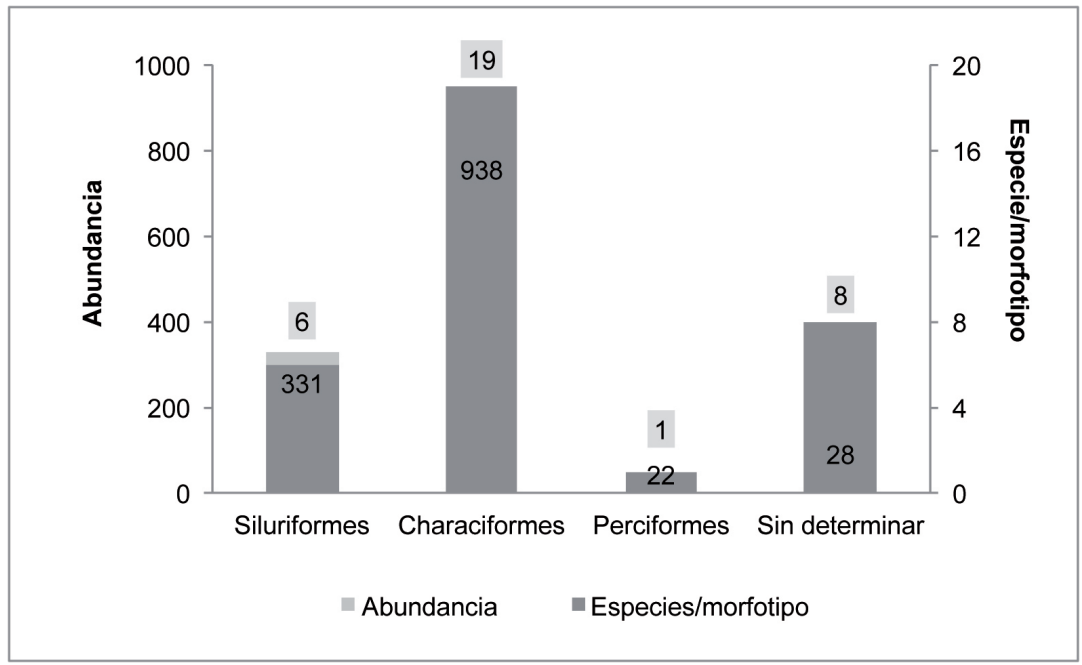

Figura 2. Abundancia de individuos y número de especies/morfotipos que conforman el ictioplancton de acuerdo al orden taxonómico. El número de especies/morfotipos se encuentra dentro del cuadro.

Las densidades oscilaron entre 0,29 y 576 individuos $/ \mathrm{m}^{3}$. La especie Astyanax sp. registró la mayor densidad, seguido de $P$. blochii, "Characiforme 7 " y "Characiforme $4 "$.

En las estaciones próximas a la influencia del río San Jorge (Caño Grande y Ciénaga-Río) (Figura 3) fueron registradas las mayores densidades, y estas a su vez presentaron diferencias entre los meses de muestreo, registrando la mayor densidad en el mes de junio con 131,4 individuos $/ \mathrm{m}^{3}$; en los meses de febrero, septiembre, noviembre se registró una densidad promedio de 1,8 individuos $/ \mathrm{m}^{3}$.

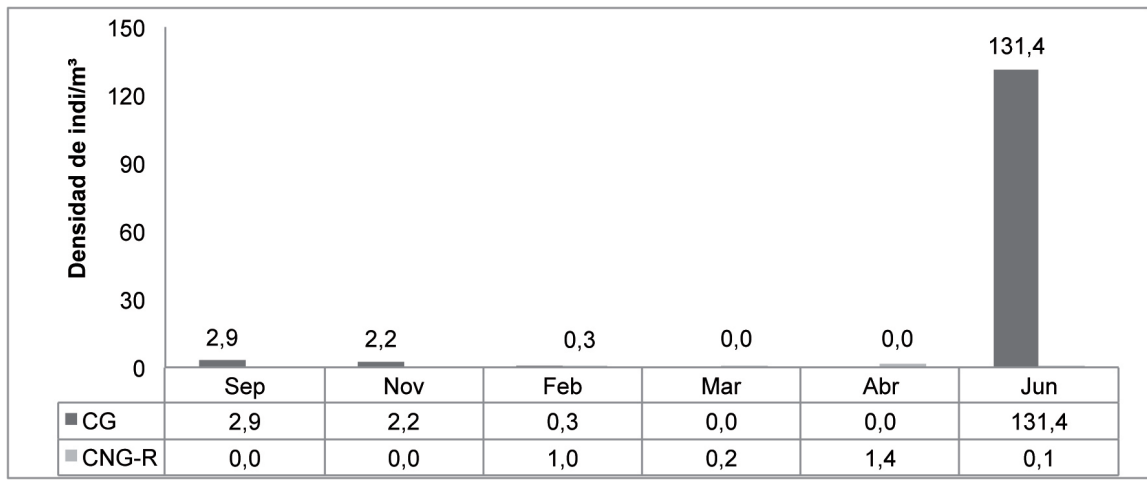

Figura 3. Densidades de ictioplancton en cada momento de muestreo en las estaciones Caño Grande (CG) y Ciénaga-Río (CNG-R). 
Del total de individuos capturados, el $0,61 \%$ se encontraban en periodo embrionario, 51,36\% en fase larval-vitelino, 26,21\% en fase de preflexión, 21,44\% en fase de flexión y $0,23 \%$ en fase de posflexión. La fase de flexión dominó dentro de los siluriformes, mientras que en los characiformes la fase larval-vitelino fue la más abundante.

La densidad de individuos estuvo relacionada con el nivel y la conductividad del agua del río San Jorge. La mayor densidad fue observada en los meses en que se observó disminución en la conductividad (Figura 4) e incremento en el nivel del agua (Figura 5) debido al ingreso del río a la Ciénaga de Ayapel.

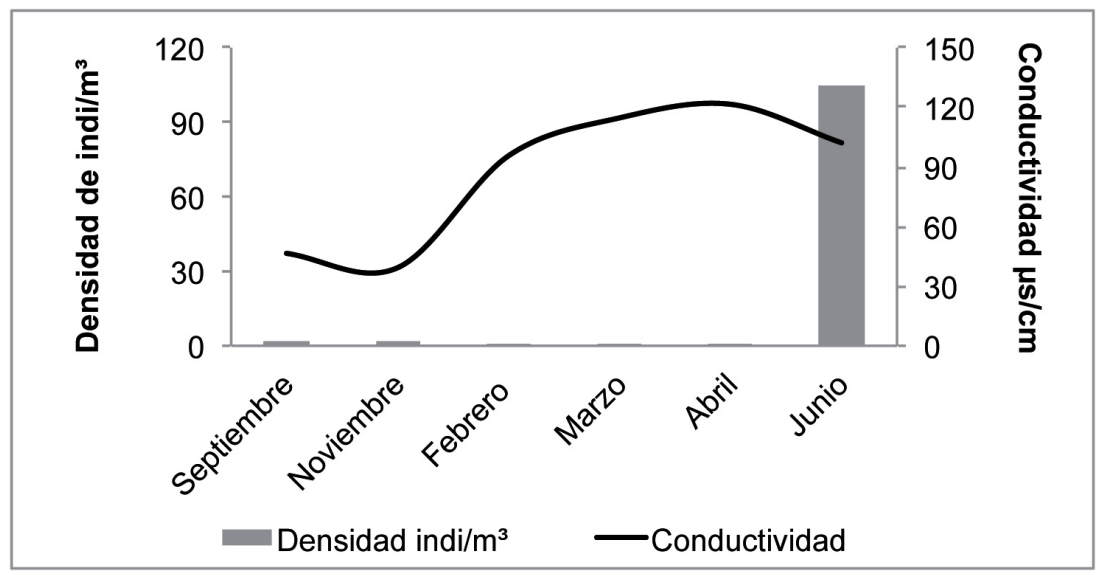

Figura 4. Densidad de ictioplancton y conductividad durante el periodo de muestreo.

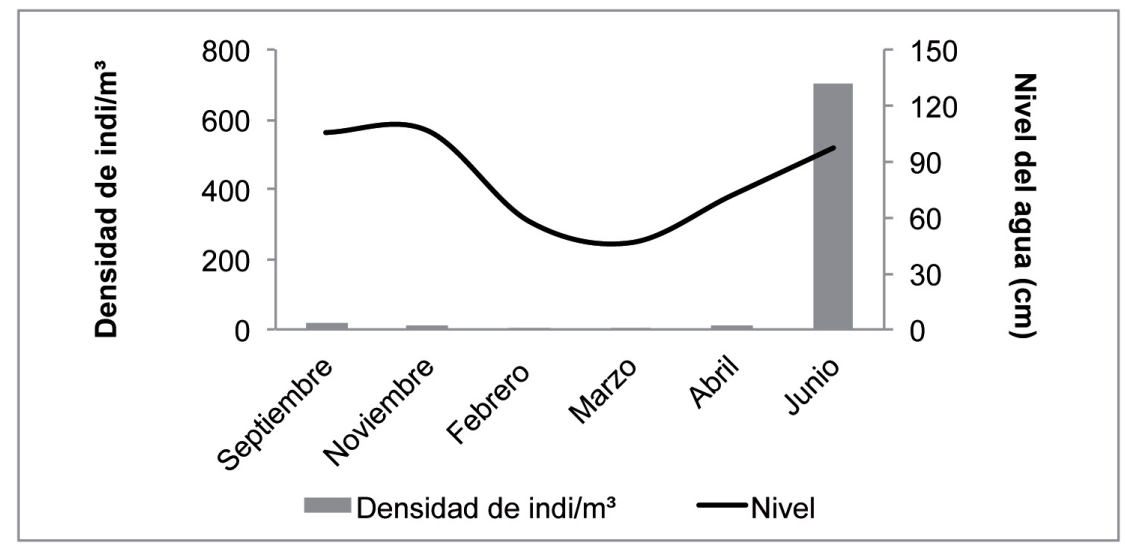

Figura 5. Densidad de ictioplancton y cambio en el nivel del agua durante el periodo de muestreo. 
La mayor densidad por morfotipo y fase de desarrollo para la estación Caño Grande fue registrada en junio y estuvo representada por Pimelodus blochii(flexión), seguido de Astyanax sp. (larval-vitelino y preflexión), "Characiforme 7" (larval-vitelino) y "Characiforme 4" (larval-vitelino). En el sector de la ciénaga más cercano a la influencia de Caño Grande (Ciénaga-Río), el morfotipo con mayor densidad fue Plagioscion surinamensis (preflexión) en el mes de febrero, seguido de "Characiforme 14" (larval-vitelino) en el mes de abril.

\section{DISCUSIÓN}

La asociación de ictioplancton colectada en la Ciénaga de Ayapel está conformada por 33 formas diferentes (6 especies y 27 morfotipos), siendo los characiformes los más abundantes seguidos por los siluriformes. Este patrón ha sido identificado en las comunidades de peces en el trópico suramericano, donde los grupos predominantes en la asociación de especies corresponde a los characiformes y siluriformes (LOWE-McCONNELL, 1987), además se ha evidenciado en estudios de ictioplancton realizados en la región amazónica y en el río Paraná por BAUMGARTNER et al. (1997) y en el pantanal de Mato Grosso por SEVERI (1997).

De las especies identificadas en la asociación observada en la Ciénaga de Ayapel, buena parte de ellas (Pimelodus blochii, Pseudoplatystoma magdaleniatum, Sorubim cuspicaudus, Prochilodus magdalenae, Leporinus muyscorum) tienen comportamiento migratorio asociado con su dinámica reproductiva. Estas especies generalmente desovan en aguas abiertas del canal principal, presentando huevos y larvas pelágicos que son llevados a áreas inundadas donde continúan con su desarrollo (AGOSTINHO et al., 1993; JIMÉNEZ-SEGURA, 1998; JIMÉNEZ-SEGURA, 2007).

Las fases de desarrollo dominantes en la asociación de ictioplancton en la Ciénaga de Ayapel son particulares al orden taxonómico. En los characiformes dominó la fase larval-vitelino, y en los siluriformes las fases de flexión y posflexión. Tanto para characiformes como para siluriformes los momentos de reproducción ocurren bajo características biológicas y ambientales específicas. De acuerdo a la clasificación de patrones reproductivos propuesta por BALON $(1975,1985)$ la cual se basa en los lugares de desove, las adaptaciones de los huevos y embriones a los sitios de reproducción y el grado de cuidado parental, generalmente las especies migratorias de characiformes y siluriformes, desovan en ambientes de corrientes y durante las crecientes generadas por las lluvias. ARAUJO-LIMA (1994) afirma que los characiformes migratorios desovan principalmente en las regiones marginales de los ríos donde hay vegetación cercana, así que es posible que la dominancia de characiformes en esta fase inicial, se deba a su corto tiempo de deriva luego del momento de la fecundación, que consecuentemente ingresan al plano de inundación debido al incremento en el nivel del río.

El ingreso de ictioplancton a la Ciénaga de Ayapel está relacionado con los aportes de caudal que el río San Jorge hace a este sistema acuático durante su ciclo hidrológico, determinando la variación y estructura de las comunidades de peces (AGOSTINHO et al., 1993). El ingreso del ictioplancton a las ciénagas, se da por la interacción de factores físicos como los niveles de precipitación anuales en sus cuencas de drenaje y el consecuente aumento en el nivel del agua, el cual es 
considerado uno de los principales factores que actúa sobre las comunidades de peces tropicales (LOWE-McCONNELL, 1987; JUNK et al., 1989; VAZZOLER, 1996), además, de la temperatura, el oxígeno disuelto y la velocidad de la corriente (CAVICCHOLI, 2000; NASCIMENTO \& NAKATANI, 2006; SANCHES et al., 2006; BAUMGARTNER et al., 2008). Factores biológicos asociados a la historia de vida de los organismos tal como la distribución de los adultos, las áreas de desove, la necesidad energética y la tasa de sobrevivencia de las larvas en el plancton (WALKER et al., 1987; SHAPIRO et al., 1988) también tienen influencia en dicho proceso.

En este sentido, la reproducción de muchas especies de peces tropicales generalmente esta sincronizada con la estacionalidad ambiental, la cual garantiza condiciones favorables para la sobrevivencia de larvas y huevos (BAUMGARTNER et al., 1997; NAKATANI et al., 1997; SEVERI, 1997). Como se observó en este estudio, la diferencia en las densidades de ictioplancton entrando a la Ciénaga de Ayapel de acuerdo con el mes, sugiere que la mayor actividad reproductiva de las especies sucede en el mes de junio y con menor intensidad en los meses de septiembre y noviembre. Especies como Pimelodus blochii y Pseudoplatystoma magdaleniatum, y algunos Characidos como Astyanax sp., Prochilodus magdalenae, Triportheus magdalenae y Leporinus muyscorum, al igual que los morfotipos Characiforme 7 y Characiforme 4, se reproducen en los meses de crecientes en el río San Jorge; en tanto, la prole ingresa a la Ciénaga de Ayapel en los momentos de mayor influencia de este río sobre su plano inundable, momento en el que ocurre un aumento considerable en el nivel del agua y cambios importantes en parámetros fisicoquímicos como la conductividad, la cual ya ha sido reportada en diversos estudios como una señal sincronizadora de los eventos de desove en ríos tropicales (NAKATANI et al., 1993; VAZZOLER, 1996; BAUMGARTNER et al., 1997; BAUMGARTNER et al., 2004; BIALETZKI et al., 2004).

Embriones, larvas y huevos de diferentes especies migratorias llegan a las áreas inundadas donde el agua que ingresa genera la descomposición del material vegetal que creció durante la temporada de estiaje y del material orgánico que ha sido arrastrado por el río, el cual se comporta como una fuente de nutrientes y materia orgánica (JUNK et al., 1989; GALLO et al., 2003); generando el incremento de nutrientes y microhábitats, que ofrecen alimento y refugio necesario para el desarrollo de los individuos en sus estados iniciales de desarrollo. La entrada de estos nuevos individuos al sistema cenagoso de Ayapel durante los meses de crecientes y aguas altas (septiembre, noviembre y junio) evidencia, por un lado, la relación existente entre la variación físico-química asociada a estos momentos hidrológicos y la reproducción de los individuos, además de la importancia de la conectividad río-plano de inundación, que facilita la llegada de las larvas y huevos por medio de la corriente a las zonas de crianza (HERGENRADER et al., 1982; PAVLOV, 1994; ARAUJO-LIMA \& OLIVEIRA, 1998; PETRY et al., 2003; BIALETZKI et al., 2004).

En relación a los ambientes, aquellos que presentaron menor complejidad de hábitat (cobertura de macrófitos) dentro de la Ciénaga de Ayapel registraron la mayor densidad de ictioplancton en los meses de mayor abundancia. La complejidad de los hábitats puede influir sobre la efectividad de los aparejos de muestreo (NAKATANI et al., 2001). Es posible que la baja densidad de ictioplancton en ambientes dentro de la Ciénaga de Ayapel se deba a que una vez las larvas entran dentro de la ciénaga 
se distribuyen bajo las zonas cubiertas por macrófitos (en búsqueda de protección y alimento), ambientes donde la red de colecta no tiene acceso.

De acuerdo a los datos obtenidos, podemos afirmar que el pulso de inundación del río San Jorge y el grado de conectividad de este plano de inundación son factores determinantes en el ingreso del ictioplancton a la Ciénaga de Ayapel, la cual a su vez se comporta como una zona de crianza de gran cantidad de especies, entre ellas algunas de interés comercial en las pesquerías locales y regionales.

\section{AGRADECIMIENTOS}

Esta investigación fue realizada gracias al apoyo de una beca del Instituto Colombiano para el Desarrollo de la Tecnología "Francisco José de Caldas", COLCIENCIAS, otorgada al Grupo de Investigación GAIA. Los autores agradecen igualmente a la fundación Idea Wild y al Fondo Internacional para el Apoyo de la Ciencia por aportar los equipos de campo. Y, muy especialmente, al Grupo de Ictiología de la Universidad de Antioquia -GIUA- por su acompañamiento en todas las fases de esta investigación.

\section{BIBLIOGRAFÍA}

AGOSTINHO, A.A., VAZZOLER, A.E.A.M., GOMES, L.C. \& OKADA, E.K., 1993.- Estratificación espacial y comportamiento de Prochilodus scrofa en distintas fases del ciclo de vida, en la planicie de inundación del alto río Paraná y embalse de Itaipú, Paraná, Brasil. Rev. Hydrobiol. Trop., 26: 79-90.

ARAUJO-LIMA, C.A.R.M., 1994.- Egg size and larval development in Central Amazonian fish. J Fish Biol., 44: 371-389.

ARAUJO-LIMA, C.A.R.M., \& OLIVEIRA, E.C., 1998.- Transport of larval fish in the Amazon. J Fish Biol, 53: 297-306.

ARAUJO-LIMA, C.A.R.M., SILVA, V.V. DA., PETRY, P., OLIVEIRA, E.C. \& MOURA, S.M.L., 2001.- Diel variation of larval fish abundance in the Amazon and Rio Negro. Braz. J. Biol., 61 (3): 1519-698.

BALON, E.K., 1975.- Reproductive guilds of fishes: a proposal and a definition. J Fish Res Board Can., 32 (6): 821-864.

1985.- Early life histories of fishes: New development, evolutionary perspectives. Dr. W. Junk Publisher, Dordrecht.

BAUMGARTNER, G., NAKATANI, K., CAVICCHIOLI, M. \& BAUMGARTNER, M.S.T., 1997.- Some aspects of the ecology of fish larva in the floodplain of high Paraná River., Brazil. Rev Bras Zool, 14:551-563.

BAUMGARTNER, G., NAKATANI, K., GOMES, L.C., BIALETZKI, A., SANCHES, P.V. \& MAKRAKIS, M.C., 2004. Identification of spawning sites and natural nurseries of fishes in the upper Paraná River. Brazil. Environ Biol Fish., 71: 115-125.

., 2008.- Fish larvae from the upper Paraná River: do abiotic factors affect larval density? Neotrop Ichthyol., 6 (4): 551-558.

BIALETZKI, A., NAKATANI, K., SANCHES, P.V. \& BAUMGARTNER, G., 2002.- Spatial and temporal distribution of larvae and juveniles of Hoplias aff. malabaricus (Characiformes, Erythrinidae) in the Upper Paraná River floodplain. Brazil. Braz. J. Biol., 62 (2): 1519-6984.

, 2004.- Eggs and larvae of the "curvina" Plagioscion squamosissimus (Heckel, 1840) (Osteichthyes, Sciaenidae) in the Baía River, Mato Grosso do Sul State, Brazil. J Plankton Res., 26 (2): 1327-1336.

BIALETZKI, A., SANCHES, P.V., CAVICCHIOLI, M., BAUMGARTNER, G., RIBEIRO, R.P. \& NAKATANI, K., 1999. Drift of ichthyoplankton in two channels of the Paraná River, between Paraná and Mato Grosso do Sul States, Brazil. Brazilian Archives of Biology and Technology, 42: 53-60.

CAVICCHIOLI, M., 2000.- Mudanças ontogênicas na morfologia do trato digestório e na dieta, e na seletividade alimentar de larvas de peixes do reservatório de Itaipú - río Paraná, Brasil: Tesis de Doctorado, Universidade Estadual de Maringá, Maringá, Brasil.

FUENTES, C.M. \& ESPINACH ROS, A. 1998.- Variación de la actividad reproductiva del sábalo, Prochilodus lineatus (Valenciennes, 1847), estimada por el flujo de larvas en el río Paraná inferior. Natura Neotropicalis, 29(1): 25-32.

GALLO, E.L., AHEARN, D., DAHLGREN, R.A. \& GROSHOLZ, E., 2003.- River-floodplain Hydrologic Connectivity: Impact on Temporal and Spatial Floodplain Water Quality and Productivity Patterns. American Geophysical Union, Fall Meeting. 
GONZÁLEZ, A.O., ROUX, J.P., HERNÁNDEZ, D.R. \& SÁNCHEZ, S., 2010.- Variación espacio-temporal del ictioplancton en un tramo del río Paraná. Interciencia, 35 (12).

HERGENRADER, G.L., HARROW, L.G., KING, R.G., CADA, G.F. \& SCHLESINGER, A.B., 1982.- Larval fishes in the Missouri River and the effects of entrainment: 185-223 (in) HESSE, L.W., HERGENRADER, G.L., LEWIS, H.S., REETZ, S.D. \& SCHLESINGER A.B. (eds.) The Middle Missouri River. The Missouri River Study Group, Missouri.

IGAC., 1986.- Estudio general de suelos de los municipios de Ayapel, Buenavista, Planeta Rica y Pueblo Nuevo (Departamento de Córdoba). Instituto Geográfico "Agustín Codazzi”, Subdirección Agrológica, Bogotá.

JIMÉNEZ-SEGURA, L.F., 1998.- Aspectos diferenciales de las comunidades de peces en grandes ríos tropicales y sus lagunas marginales. Seminario de ecología de comunidades. Universidad Federal de Minas Gerais. Belo Horizonte (Brasil).

JIMÉNEZ-SEGURA, L.F., 2007.- Aspectos reproductivos de peces migradores en la cuenca del río Magdalena: Tesis, Universidad de Antioquia, Instituto de Biología, Medellín.

JUNK, W.J., BAYLEY, P.B. \& SPARKS, R.E., 1989.- The Flood pulse concept in river floodplain systems. Canadian Special Publication of Fisheries and Aquatic Sciences.

KENDALL, A.W., AHLSTROM, E.H. \& MOSER. H.G., 1984.- Early life history stages of fishes and their characters (in) MOSER, G., RICHARDS, W.J., COHEN, D.M., FAHAY, M.P., KENDALL, A.W. \& RICHARDSON, S.L. (eds.) Ontogeny and systematics of fishes. Amer. Soc. Ichthyol. Herpet. Spec. Publ, 1: 11-22.

KONDOLF, G.M., BOULTON, A.J., O'DANIEL, S., POOLE, G.C., RAHEL, F.J., STANLEY, E.H. et al., 2006.- Processbased ecological river restoration: visualizing three-dimensional connectivity y dynamic vectors to recover lost linkages. Ecol Soc1, 1(2): 5.

LEGENDRE, L. \& DEMERS, S. 1984.- Towards Dynamics Biological Oceanography and Limnology. Can. J. Fish Aquat. Sci, 41: 2-19.

LOWE-McCONNELL, R.H., 1987.- Ecological studies in tropical fish communities. Cambridge Tropical Biology Series. Cambridge Press, Cambridge.

LUCAS, M. \& BARAS, E., 2001.- Migration of freshwater fishes. Blackwell Science, Nueva York.

MACKAS, D.L., DENMAN, K.L. \& ABBOT, M.R., 1985.- Plankton patchiness: Biology in the physical vernacular. Bull. Mar. Sci., 37 (2): 652-674.

MINISTERIO DEL MEDIO AMBIENTE \& INSTITUTO DE INVESTIGACIONES DE RECURSOS BIOLÓGICOS ALEXANDER VON HUMBOLDT., 1999.- Humedales Interiores de Colombia: Bases Técnicas para su Conservación y Uso Sostenible. Bogotá.

NAKATANI, K., AGOSTINHO. A.A., BAUMGARTNER, G., BIALETZKI, A., SANCHES, P.V., MAKRAKIS, M.C. \& PAVANELLI, C.S., 2001.- Ovos e larvas de peixes de água doce: desenvolvimento e manual de identificação. Maringá, Eduem.

NAKATANI, K., BAUMGARTNER, G. \& CAVICCHIOLI, M., 1997.- Ecologia de ovos e larvas de peixes: 201-306 (en) VAZZOLER, A.E.A. DE M., AGOSTINHO, A.A. \& N. S. HAHN, N.S. (eds.) A planície de inundação do alto Rio Paraná: Aspectos físicos, biológicos e econômicos. Maringá, Eduem.

NAKATANI, K., LATINI, J.D., BAUMGARTNER, G. \& BAUMGARTNER, M.S.T., 1993.- Distribuição espacial e temporal das larvas de curvina (Plagioscion squamosissimus Heckel, 1984) (Osteichthyes, Scianidae), no reservatório de Itaipú. Revista UNIMAR, 15: 1991-209.

NARANJO, A. \& PONCE, L., 1999.- Libro Humedales Interiores de Colombia: Bases técnicas para su conservación y uso sostenible. Instituto de Investigación de Recursos Biológicos Alexander von Humboldt, Bogotá.

NASCIMENTO, F.L. \& NAKATANI, K., 2006.- Relações entre fatores ambientais e a distribuição de ovos e larvas de peixes na sub-bacia do rio Ivinhema, Estado de Mato Grosso do Sul, Brasil. Acta Sci., 28 (2): 117-122.

OLDANI, N.O., 1990.- Variaciones de la abundancia de peces del valle del río Paraná. Rev D’Hydrobiologíe Trop, 23(1): 67-76.

OLDANI, N.O., IWASZKIW, J., PADÍN, O. \& OTAEGUI, A., 1992.- Fluctuaciones de la abundancia de peces en el alto Paraná (Corrientes, Argentina). Actas del II Seminario El río Uruguay y sus recursos, 1(1): 43-53.

PAREJA-CARMONA, M.I., JIMÉNEZ-SEGURA, L.F. \& OCHOA-ORREGO, L.E., 2014.- Variación espacio-temporal de las larvas de tres especies de peces migratorios en el cauce del río Magdalena (Colombia), durante el ciclo hidrológico 2006-2007. Actual Biol, 36: 33-38.

PAVLOV, D.S., 1994.- The downstream migration of young fishes in rivers: mechanisms and distribution. Folia Zool., 43: 193-208.

PETRY, P., BAYLEY. P.B. \& MARKLE, D.F., 2003.- Relationships between fish assemblages, macrophytes and environmental gradients in the Amazon River floodplain. J Fish Biol, 63(3): 547-579.

RYNALTE-TATAJE, A., NAKATANI, K., FERNANDES, R., AGOSTINHO, A.A., BIALETZKI, A., 2011.- Temporal distribution of ichthyoplankton in the Ivinhema River (Mato Grosso do Sul State/Brazil): Influence of enviromental variables. Neotrop Icthyol 9(2):427-436

RESTREPO, C.A., 2005.- Aproximación a la dinámica del transporte del nitrógeno y el fósforo en la ciénaga de Ayapel: Tesis, Universidad Nacional de Colombia, Medellín.

RÍOS, M.I., 2006.- Variación espacio-temporal en la asociación de peces en el sistema cenagoso de Ayapel, Córdoba (Colombia) durante el ciclo hidrológico 2004-2005: Tesis, Universidad de Antioquia, Medellín.

SALETE, V., GOGOLA, T.M., VANDERLEI, P., BAUMGARTHER, G., BAUMGARTNER, D., PIANA, P. et al., 2009.Fish larvae assemblages in two floodplain lakes with different degrees of connection to the Paraná River, Brazil. Neotrop Ichthyol., 3: 429-438. 
SANCHES, P.V., NAKATANI, K., BIALETZKI, A., BAUMGARTNER, G., GOMES, L.C. \& ANTONIASSI, E., 2006.Flow regulation by dams affecting ichthyoplankton: the case of the Porto Primavera Dam, Paraná River, Brazil. River Res Appl., 22: 555-565.

SEVERI, W., 1997.- Ecologia do ictioplâncton no pantanal de Barão de Melgaço, bacia do rio Cuiabá, Mato Grosso, Brasil: Tesis, Universidade Federal de São Carlos, São Carlos.

SHAPIRO, D.Y., HENSLEY, D.A. \& APPELDOORN, R.S., 1988.- Pelagic spawning and egg transport in coral reef fishes: a skeptical overview. Environ Biol Fish., 22 (1): 3-14.

VAZZOLER, A.E.A.M., 1996.- Biologia da reprodução de peixes teleósteos: teoria e prática. Editorial de la Universidad Estadual de Maringá, Maringá.

WALKER, H.J. JR, WATSON, W. \& BARNETT, A.M., 1987.- Seasonal occurrence of larval fishes in the nearshore southern California Bight off San Onofre, California. Est Coast Shelf Sci, 25: 91-10.

WELCOMME, R.L., 1979.- Fishery management in large rivers. FAO Fisheries Technical Paper 194. Roma, Italia. 1985.- River fisheries. FAO Fisheries Technical Paper 262. Roma, Italia.

WINEMILLER, K.O., 1998.- Floodplain river food webs: Generalizations and implications for fisheries management. University Texas A\&M, Department of Wildlife and Fisheries Sciences. 